\title{
HOME MEDICINE REVIEW ELDERLY BASED ASSESSMENT OF MEDICATION RELATED PROBLEMS IN MYSURU CITY
}

\author{
JAIDEV KUMAR, MAHENDRAPPA*, PRAMOD KUMAR \\ Department of Paediatric, JSS Medical College and Hospital, SS. Nagar, Mysore 570015 \\ Email: kbmahenappa@jssuni.edu.in
}

Received: 15 Apr 2019, Revised and Accepted: 13 Jun 2019

\begin{abstract}
Objective: To assess the medication related problems among chronically ill patients.
\end{abstract}

Methods: Patients who met study criteria were enrolled in this research study, followed by standard operating procedure of home medicine review was applied to carry out this research study. Drug related problems of enrolled patients were evaluated by applying Hepler and Strand drug related problem scale.

Results: The pharmacist intervention rate in this research study was found $58 \%$. The medication related problems identified in this study were drug given without indication which was accountable for $32 \%$ followed by drug duplication $5.21 \%$, drug interactions $11 \%$ and adverse drug reactions $6.52 \%$.

Conclusion: This research study concludes that prevalence of drug related problems among elderly patients were accountable for $40.49 \%$ among Home Medicine Review in Mysore city due to more comorbidity which had resulted more number of medications with respect to individual medical case. Elderly patients will have lot of confusion which makes difficult to follow strict directions of medications prescribed by concerned consultant.

Keywords: Home, Medicine, Review

(c) 2019 The Authors. Published by Innovare Academic Sciences Pvt Ltd. This is an open access article under the CC BY license (http://creativecommons.org/licenses/by/4.0/) DOI: http://dx.doi.org/10.22159/ijcpr.2019v11i4.34940

\section{INTRODUCTION}

Patients with medication-related problems will not be able to achieve the correct therapeutic endpoint which can ultimately result in further progression of disease and complications of disease. The common risk factors of medication-related problems are such as increased co morbidities, hepatic impairment, renal impairment, lack of certain biological enzymes such as glucose 6 phosphate dehydrogenase, the safety of medications that needs to be considered in specialised population such as pregnant and lactating women followed by infants, children, and elderly persons. Any patient with more comorbidities will always be prescribed with more number of medications where each and every medication needs to be screened for medication related problems such as drug duplication will be labelled for drug found in any prescription if it meets following where two different brand names were prescribed having the same generic combination, drug given without indication, drug not given when there is indication, under dosing, overdosing, adverse drug reactions, drug interactions. Elderly patients suffering from any chronic medical problems needs to have an assessment of hepatic and renal function status because most of the medications usually undergo metabolism in the liver followed by excretion in kidneys. Any patient with hepatic and renal impairment needs to have hepatic and renal dosage adjustments, failure to do hepatic and renal dosage adjustments can always result toxic dose to these patients under these circumstances where the patient may not be able to achieve therapeutic endpoint in according to the anticipated goal of treatment. A medication with narrow therapeutic index' such as digoxin, theophylline, lithium etc needs to have close monitoring for these medications to evaluate dosing regimen, adverse drug reactions and drug interactions. Elderly patients due to advanced age the physiology of organs naturally declines which makes more sensitivity for each and every medication. Patients receiving theophylline needs to have close monitoring for drug drug interactions as there are plenty of drug interactions with theophylline and it also needs to evaluate adverse drug reactions.

The prevalence of medication use increases among elderly population as the age progresses with the statistics reflecting that people aged more than $65 \mathrm{y}$ use at least one medication per week which is accountable for $90 \%$ whereas more than $40 \%$ of elderly population utilise at least five different medications/week and $12 \%$ of the elderly population utilise more than 10 different medications per week
Female gender will be receiving more number of medications compared to male for the following therapeutic indications such as psychoactive and arthritic disorders. Research author suggests that medication use among the elderly population was more among weak people, inpatients and patients in old age care homes. Patients residing in old age care homes will be receiving seven to eight different medications as per research investigator findings [1].

The research findings of drug-related problems are commonly observed among elderly patients falling in the age range of $60 \mathrm{y}$ and above. A drug-related problem has been defined as "an event or circumstance involving drug treatment that actually or potentially interferes with a patient's experiencing an optimum outcome of medical care. Therapeutic failure may happen when most of the medications are underutilised as per the standard recommendations of drug monograph, as well as with other reasons such as not following the directions by patient given by clinician as well as prescribing lower strength of generic drug by the prescriber. Older research studies have shown that $15-22 \%$ of hospitalised elderly patients happens due to drug-related problems. According to this research author majority of adverse drug, reactions can be prevented and the maximum of elderly patients who got admitted in hospitals have drug-related problems. Drugs prescribed without indications among hospitalised elderly patients are commonly observed and $25 \%$ of total drug-related problems are accountable for drug given without indication. Severe drug-related problems will always have life-threatening reactions among elderly patients and if not treated at the right time, the prognosis of hospitalisation could be very bad among elderly patients which can also have worst impact on mortality of elderly patients. According to one research statistics adverse drug reaction is the fourth leading cause of death in USA. One of the research work carried out in USA reflects that every dollar charged on purchasing drugs in US nursing home facilities, 1.33 dollars in health care resources were utilised in the management of drug-related problems. Expenditure fetched for treating drug-related problems among elderly patients can result economic burden and can cause an economic burden to the society as per this research investigator findings [2].

India is a country with more than one billion populations and with more than one lakh formulations available in the pharmaceutical markets, where there is a possibility of finding the potential for drug-related problems [3]. The use of irrational combinations, lack of evidence-based 
medicine practices and illiteracy among Indian patient population contributes to medicine-related problems. Today in India there are lot of drug-related problems which goes unnoticed as most of the medical case sheets in hospitals are not properly screened due to lack of clinical pharmacy services which do not exist in most of the hospitals.

Elderly patients suffering from more than one co morbities will always have more number of medications prescribed by prescriber where they may have one or more drug-related problems if the prescription is irrational as well as due to the aging problem [4]. Elderly patients suffering from chronic illness finding very difficult to come for follow up to clinic when their respective home is too far away from the destination of health care institution such as a hospital or private clinic needs a health care professional to assess the prognosis of elderly patients in these circumstances [5]. Home Medicine Review among these elderly patients can have better prognosis which was reflected in older research studies carried out in Australia and England [6]. In India Home Medicine Review research work carried out was very less as far the research investigators knowledge and therefore research investigators designed this research protocol in the interest of providing better health care services to elderly persons suffering from chronic illness as well as to clinicians practicing in and around the Mysuru city. The proposed study aims to gather information on medicine-related issues in the community setting in the context of the HMR process and evaluate the impact of HMR associated drug-induced morbidity and mortality.

\section{MATERIALS AND METHODS}

\section{General objective}

To initiate and evaluate the Home Medicine Review services in Mysore city.

\section{Study objective}

To assess the medication-related problems among chronically ill patients.

\section{Methodology}

This research study design was cross-sectional observational and interventional study carried out over a period of one year in Mysore city. Study participants who meet study criteria were enrolled into this study and information was documented in the suitably designed data collection form. Demographic details of subject participants such as age, gender, qualification status, marital status etc were documented in the suitably designed data collection form. HMR study was initiated in Mysore city by selecting ten general practitioners practicing in ten different locations of Mysore city. Patient present and past medical record were reviewed to find out whether a patient is properly following the directions given by the concerned consultant. During medication review, different aspects were reviewed such as medication adherence, medication-related problems, an alternative system of medicine, and storage of medicine, history of over the counter medications, medication errors and allergic history of medications. Investigators of this research study were able to explain the importance and benefits of HMR to ten general practitioners practicing in Mysore city and in according to their willingness to participate they were enrolled in this research study. HMR referral form was designed and given to selected GP for their referral of patients meeting study criteria. Upon GP referral, the investigator was scheduling a suitable time of patient by taking appointment of the patient through telephone before conducting HMR interview. During HMR interview, patient data was documented in suitable HMR referral form that was designed and the same was communicated to refer GP. If there are any issues with regarding to found drug therapy in that particular case it was intimated to referred GP and upon GP consensus certain remedial action was taken.

\section{RESULTS AND DISCUSSION}

Table 1: Demographic details of the Subjects, total number of subjects enrolled was-568

\begin{tabular}{|c|c|c|c|}
\hline S. No. & Gender & Total number & Percentage \\
\hline 1 & Male & 388 & 68.30 \\
\hline 2 & Female & 180 & 31.69 \\
\hline \multicolumn{4}{|c|}{ Qualification status of enrolled subjects } \\
\hline 3 & Illiterate & 170 & 29.92 \\
\hline 4 & I Standard-V Standard & 23 & 04.04 \\
\hline 5 & VI Standard-X Standard & 145 & 25.52 \\
\hline 6 & SSLC & 95 & 16.72 \\
\hline 7 & II PUC & 65 & 11.44 \\
\hline 8 & Degree & 46 & 08.09 \\
\hline 9 & Post Graduate Degree & 22 & 03.87 \\
\hline 10 & $\mathrm{PhD}$ & 2 & 0.35 \\
\hline \multicolumn{4}{|c|}{ Age range of enrolled subjects } \\
\hline 11 & $60-70 \mathrm{Y}$ & 213 & 37.5 \\
\hline 12 & $71-80 \mathrm{Y}$ & 288 & 50.70 \\
\hline 13 & $81-90 \mathrm{Y}$ & 65 & 11.44 \\
\hline 14 & $91-100 \mathrm{Y}$ & 02 & 0.35 \\
\hline \multicolumn{4}{|c|}{ Marital status of enrolled subjects } \\
\hline 15 & Male with married & 558 & 98.23 \\
\hline 16 & Male without married & 08 & 01.40 \\
\hline 17 & Female with married & 562 & 98.94 \\
\hline \multirow[t]{2}{*}{18} & Female without married & 03 & 0.52 \\
\hline & Widow & 01 & 0.17 \\
\hline \multicolumn{4}{|c|}{ Social history of enrolled subjects } \\
\hline 19 & Male Smoker & 145 & 25.52 \\
\hline 20 & Male without Smoker & 423 & 74.47 \\
\hline 21 & Female Smoker & Nil & 00 \\
\hline 22 & Female without Smoker & 568 & 100 \\
\hline 23 & Male with Alcoholism & 48 & 08.45 \\
\hline 24 & Male without Alcoholism & 520 & 91.54 \\
\hline 25 & Female with Alcoholism & 03 & 0.52 \\
\hline 26 & Female without Alcoholism & 566 & 99.64 \\
\hline
\end{tabular}

Table 2: Feedback given by patients during home medicine review

\begin{tabular}{ll}
\hline S. No. & Comments of patients \\
\hline 1. & Few patients expressed that this concept was not known till today. \\
2. & Patients with higher literacy rate have expressed that there is certainly role of pharmacists for creating awareness regarding medications. \\
3 & Some patients have expressed there is no role of pharmacist when patient medical condition is stable. \\
4 & One of the health caretaker of patient expressed that this type health care service should be given to poor patient, without any valid reason. \\
5 & Few patients were extremely happy for providing information regarding disease and medication aspects. \\
6 & Most of the patients trust more on doctors than pharmacists. \\
7 & Some of the patients refused to give permission for home visit, as they were worried whether pharmacist are strangers. \\
8 & Some patients expressed that it can disturb their privacy. \\
\hline
\end{tabular}


Table 3: Medication issues found during home medicine review

\begin{tabular}{ll}
\hline S. No. & Medication issues \\
\hline 1 & Insulin was stored in a plastic box, rather than in the refrigerator in controlled temperature i. e 2-8 degree centigrade. \\
2 & Few antihypertensives and antidiabetic medications were expired two months back, but the patient was receiving. \\
3 & Few patients did not gargle with warm water after receiving long-acting beta two agonists and corticosteroid combination which had \\
& resulted in oral candidiasis. \\
4 & One patient took more pain killers, as the patient was suffering from neuropathic pain. \\
5 & Change of administration sites was not known among the few patients those who were receiving insulin. \\
6 & Two patients were instilling eye drops in spite of experiencing more itching and irritation after administration. \\
& Three patients were using ear drops, even after opening the container for more than one month (any eye and ear drops should be used \\
9 & within one month after opening container) \\
9 & Few patient had swallowed mouth wash, instead of spitting out medicine.
\end{tabular}

\section{Prevalence of drug-related problems}

The prevalence of drug-related problems was calculated by applying the following formula.
Drug-Related Problems Found among patients/Total number of enrolled patients $\times 100$

$230 / 568 \times 100=40.49 \%$

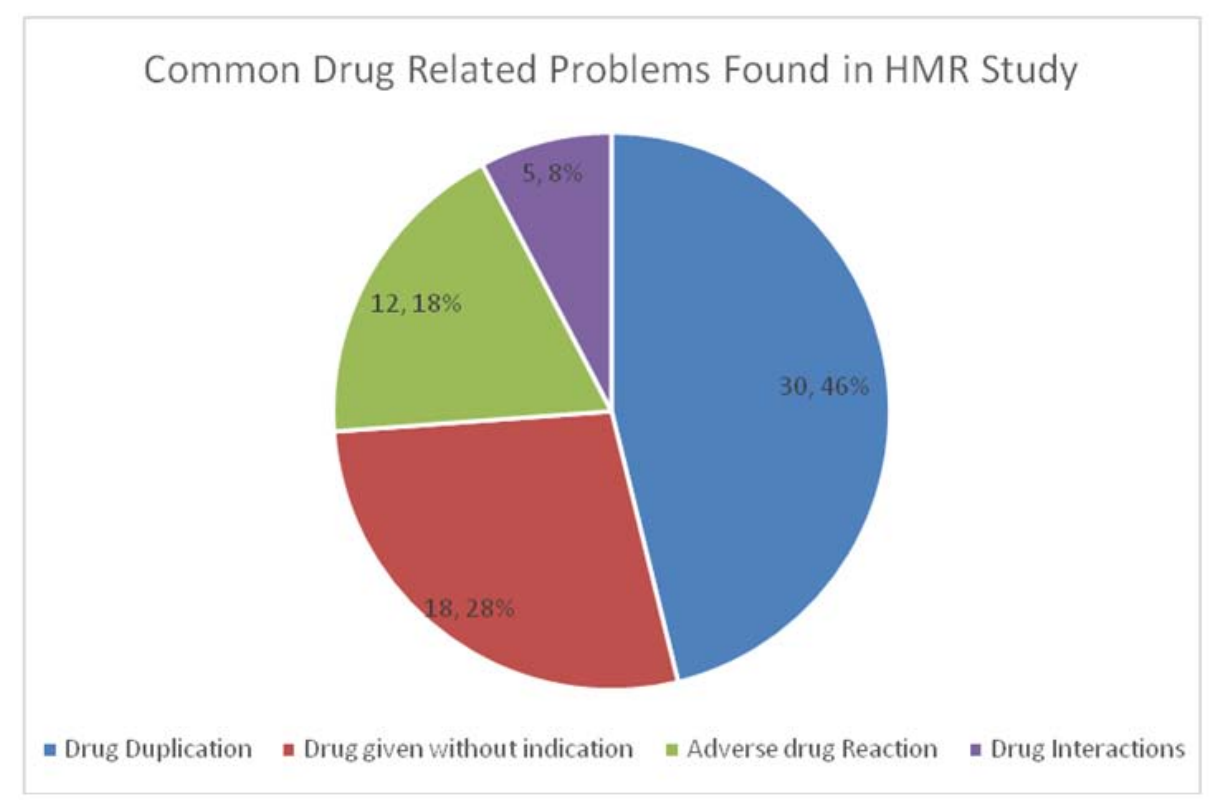

Fig. 1: Common drug-related problems found in HMR study

Table 4: The common medications that were found to show medication-related problems in this HMR study

\begin{tabular}{ll}
\hline \multicolumn{2}{l}{ Total number of medication-related problem were-230 } \\
\hline Name of medicine & Type of medication-related problem \\
\hline Amlodipine, Diclofenac & Adverse Drug Reaction-15/230 $=6.52 \%$ \\
Acleofenac+Paracetmol & Drug Duplication- $12 / 230=5.21 \%$ \\
Ranitidine & Drug given without Indication- $45 / 230=19.56 \%$ \\
Pantoprazole & Drug given without Indication-28/230 $=12.17$ \\
Cefixime, Ciprofloxacin, Metformin,Ramipril & The dose prescribed for all these medications were more than the recommended doses in renal \\
& impairment patients-25/230=10.86\% \\
\hline
\end{tabular}

Table 5: Pharmacist intervention in HMR study

\begin{tabular}{lllll}
\hline \multicolumn{2}{l}{ Total number of pharmacist intervention were-130 } & & \\
\hline S. No. & Type of intervention & Accepted intervention & Percentage & Not accepted intervention \\
\hline 1 & Adverse drug Reaction & $35 / 130$ & 26.92 & $20 / 130$ \\
2 & Drug Duplication & $12 / 130$ & 9.23 & $05 / 130$ \\
3 & Overdose & $14 / 130$ & 10.76 & $08 / 130$ \\
4 & Drug Interaction & $17 / 130$ & 13.07 & $19 / 130$ \\
\hline
\end{tabular}

According to this research study the following research findings were reflected such as The drug-related problems were grouped into 3 main categories: problems related to a home medication, problems related to a postoperative medication, and problems related to a potential indication for drug therapy Kwan and others five found that the most common postoperative problem related to home 
medications was omission of medications (46.4\%), and the most common problems related to postoperative orders involved incorrect dose and frequency. Our research study had similar findings to that of Kwan [7] and other which was reflected in fig. 1.

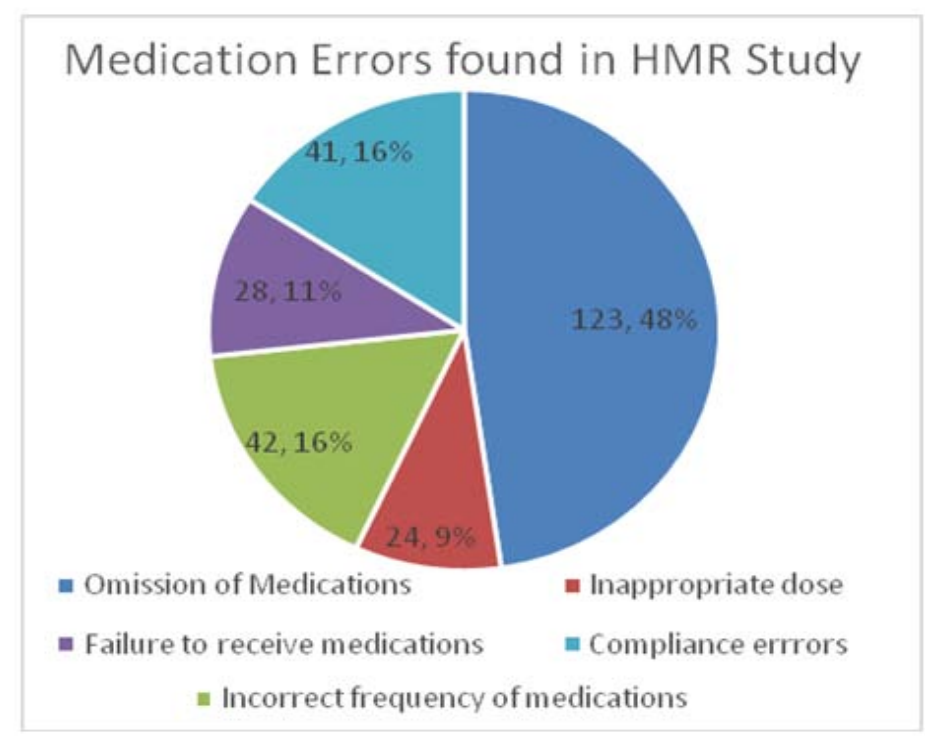

Fig. 2: Medication errors found in HMR study

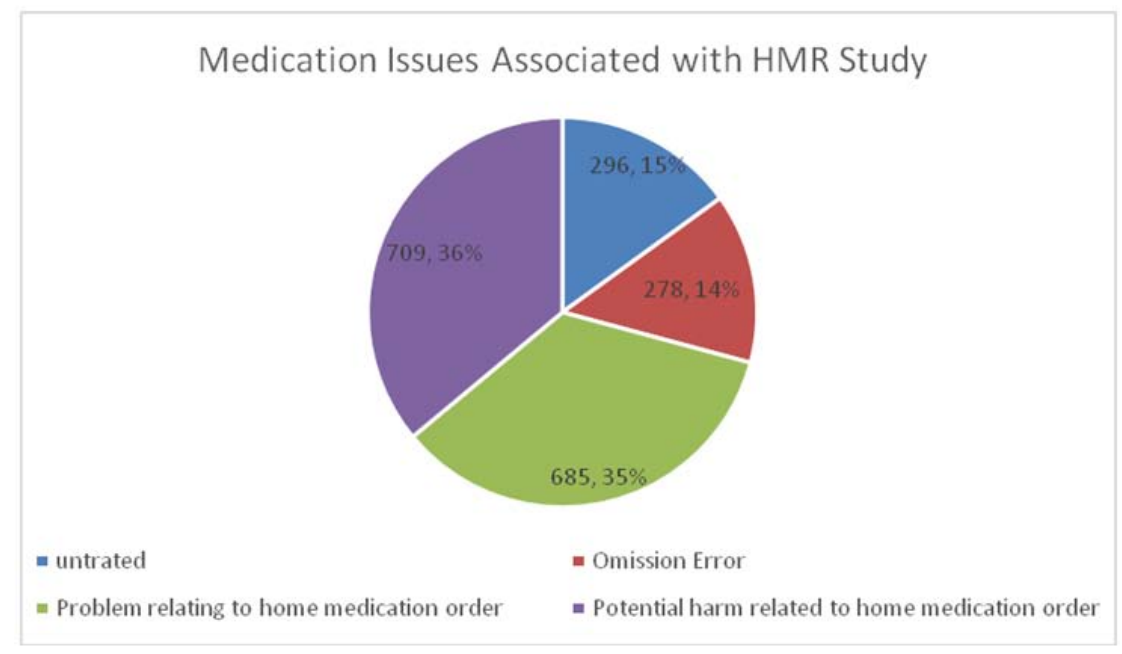

Fig. 3: Medication issues associated with HMR study

Fig. 1 depicts medication errors identified at the time of medication interview conducted at patient home. Omission of medications may have occurred due to poor prescription by a written doctor or it may not be very clear at the time of dispensing medications for dispensing pharmacist which he/she did not take any chances for dispensing medications. Compliance errors occurred in this research study was due to an expensive medicinal product, difficult administration technique followed by failure to take medications due to a busy working schedule of the patient.

Similarly, the most common types of drug-related problems in the current study were the omission of medications, illegible drug orders, inappropriate dose frequencies, and drug-allergy interactions. Thus, a focus on preventing these specific types of of medication errors could result in fewer drug-related problems and fewer health care consequences for the patient.

Cornish and others13 studied unintended discrepancies [8] between the physician's medication orderson admission and a comprehensive medication history obtained at the time of hospital admission for patients admitted to general internal medicine clinical teaching units [8]. They found that $53.6 \%$ of patients had at least one unintended discrepancy and the most common error $446.4 \%$ of errors) was the omission of regularly used medications. In addition, $38.6 \%$ of the drug-related problems identified had the potential to cause moderate to severe discomfort or clinical deterioration (as determined by consensus among evaluators). Likewise, we found that $60.3 \%$ of patients had at least one problem relating to home medication orders, with $43.3 \%$ of these problems deemed potentially harmful. Our research findings also have similar research findings to that of [8] which was reflected in fig. 2.

According to this research study [4] the following research findings were reflected, not taking/receiving a prescribed drug appropriately was the most common DRP identified (35.2\% range $4.7-49.3 \%$ ), followed by requiring drug therapy but not receiving it $(16.6 \%$, range6.9-54.5\%) and not taking/receiving the appropriate drug therapy $(14.5 \%$ range $3.6-42.6 \%)$. The least common DRP identified was a drug interaction $(1.9 \%$, range 0 $9.2 \%)^{4}$. Our research findings had similar results to that of $[9,10]$ which was reflected in fig. 3 


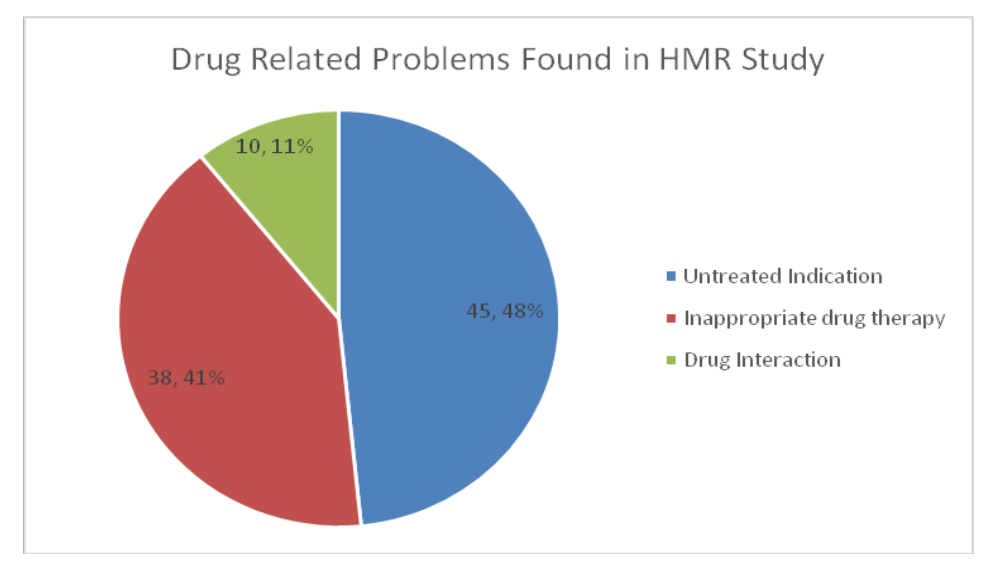

Fig. 4: Drug related problems found in HMR study

\section{CONCLUSION}

This research study concludes that prevalence of drug-related problems among elderly patients was accountable for $40.49 \%$ among Home Medicine Review in Mysore city due to more comorbidities which had resulted in more number of medications with respect to the individual medical case. Elderly patients will have lot of confusion which makes difficult to follow strict directions of medications prescribed by the concerned consultant. The situation of elderly patients can make still more miserable when no family members are living with them. Therefore in this type of situation, the role of pharmacist can play an important role in creating more awareness of medications to elderly patients. Elderly patients can be suspected with more number of medication-related problems when they have increased comorbid conditions which ultimately require more screening of medications during those situations. Failure to have a close assessment of medications among elderly patients may end up with more medication-related problems which goes unnoticed and the further proper therapeutic endpoint will not be achieved among these patients. Prognosis of any elderly patients may become very bad when there is more medication-related problems. This research study had given clear indication that any elderly patients meeting following requirements such as receiving 10 doses per day followed by staying too far away from concerned consultant, receiving prescribed medications with more than five to six medical consultants, elderly patients with no health caretaker or having dementia as major problem needs to be subjected/directed for Home Medication Review. The major medicationrelated problems identified in this study were a drug is given without indication $32 \%$ followed by drug duplication $5.21 \%$, drug interactions $11 \%$ and adverse drug reactions $6.52 \%$. The pharmacist intervention rate in this research study was found was $58 \%$.

\section{ACKNOWLEDGEMENT}

Our humble Pranamas to his holiness feet Sri Jagad guru Shiva rathri Deshikendra Mahaswami ji and all authors thank patients, doctors for cooperating to carrying out this research work in Mysore city. Without the cooperation of patients and doctors this research work would not be successful.

\section{AUTHORS CONTRIBUTIONS}

All the author have contributed equally to make this research manuscript successfully

\section{CONFLICT OF INTERESTS}

\section{Declare none}

\section{REFERENCES}

1. https://www.msdmanuals.com/professional/geriatrics/drugtherapy-in-the- [Last accessed on 18 Sep 2018]

2. Patrik Midlov, Tommy Erikkson, Annika Krogh. Drug-related problems in the elderly. Sweden: Springer. Available from: www.springer.com [Last accessed on 16 Nov 2011]

3. Lee E, Braund R, Tordoff J. Examining the first year of medicines use review services provided by pharmacists in New Zealand. J New Zealand Med Association 2008;122:1293.

4. http://www.medicareaustralia.gov.au/provider/pbs/fifthagreement/home-medicines-review.jsp). [Last accessed on 14 Dec 2013].

5. Guidelines for pharmacists providing Residential Medication Management Review (RMMR) and Quality use of Medicines (QUM) services. Pharmaceutical Society of Australia; 2011. p. 1-23.

6. Turner JP, Bell JS. Implementation of pharmacist-led medication reviews in general practice. Int J Clin Pharm 2012;12:1-2.

7. Melissa Haley, Colette Raymond, Cesilia Nishi, Eric Bohm. Drugrelated problems in patients undergoing elective total joint arthroplasty of the hip or knee. Can J Hosp Pharm 2009;5:360-6.

8. Patricia L Cornish, Sandra R Knowles, Romina Marchesano, Vincent Tam, Steven Shadowitz, David N Juurlink, et al. Etchells unintended medication discrepancies at the time of hospital admission. Arch Intern Med 2005;165:424-9.

9. Elaine Lau, Lisa R Dolovich. Drug-related problems in elderly general practice patients receiving pharmaceutical care. Int J Pharm Practice 2005;13:165-77.

10. Gowan J, Roller L. Elder abuse, medication reviews and the pharmacist. Australian J Pharmacy; 2009. p. 90. 